\title{
Investigation of Medication Errors in a Tertiary Care Hospitals in the Qassim Region, Saudi Arabia
}

\author{
Amjad Al-Harkan ${ }^{1 *}$, Njood Al-Harkan², Amal Al-Najjar ${ }^{3}$, Alaa Al-Hunti ${ }^{2}$, Ahmed Al-Rashidi ${ }^{4}$, Abdullah Al-Themery $^{5}$ \\ ${ }^{1}$ Department of Clinical Pharmacy, College of Pharmacy, Qassim University, Qassim, Saudi Arabia; ${ }^{2}$ Department of Medicine \\ and general surgery, College of Medicine, Qassim University, Qassim, Saudi Arabia; ${ }^{3}$ Drug and Poison Information Specialist, \\ Pharmacy Services, Security Forces Hospital Program, Riyadh, Saudi Arabia; ${ }^{4}$ Department of Quality Management and Patient \\ Safety, Medication Safety Officer, Maternity and Children Hospital, Qassim, Saudi Arabia
}

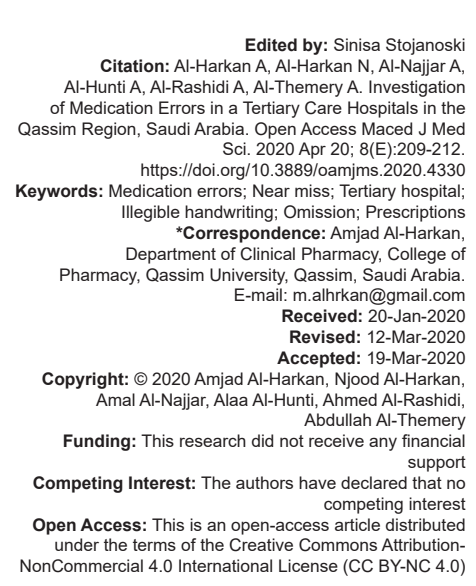

Introduction

Medication errors (MEs) occur when patients are harmed or inappropriate medication use happens due to avoidable reasons while the medication is in the possession of health-care providers or patients [1]. The definition of MEs is broad, involving different aspects of any type of medication-related issues including dosage, selection of drug, time, or method of administration, omission of prescribed medication, or the administration of a medication without a valid order [2]. Different systems exist for classifying prescription errors. For example, the Institute of Medicine has categorized three types of medical errors related to patient utilization: Underuse (failure to prescribe when benefit would be likely), overuse (prescribing when benefit is unlikely), and misuse (an actual error or mistake) [3]. Neville et al. have classified prescription errors into four categories on the basis of potential clinical outcomes, ranging from serious to patients (A) to inconsequential (D) [4].
MEs are underreported in all countries, particularly in developing countries [5]. The Institute of Medicine estimated that MEs are linked with approximately 7000 patient deaths each year [6]. Makary and Daniel (2016) have suggested that MEs are the third leading cause of death in the United States, but the issue is underreported due to the fact that the data usually focus on medical conditions rather than medical mistakes in cases of reported death [7]. In Saudi Arabia, over 40,000 medical error complaints are filed annually [8]. The rate of prescribing errors has been estimated to involve $11 \%$ of prescriptions in primary care in contrast to $1.5 \%$ in hospitals. Errors resulting in preventable adverse drug events occurred most often at the stages of ordering $(56 \%)$ or administering $(34 \%)$, whereas transcribing $(6 \%)$ and dispensing errors (4\%) were less common [9].

These errors can have profound implications for patients, families, and health-care providers. The human and societal suffering of patients experiencing costly and prolonged hospital stays can be significant, and some patients never fully recover to their premorbid 
status. Errors erode patient, family, and public confidence in health services [7]. Moreover, health care-associated errors, regardless of the type, cast a great financial burden on the health-care budget. It was estimated that errors in health care in the United States accounted for over $\$ 17$ billion in cost in 2008 [10].

Literature exploring MEs in the Kingdom of Saudi Arabia is limited and has mainly focused on the Riyadh region [1], [6]. The main goal of the study was investigation of MEs in a tertiary care hospitals in the Qassim region, Saudi Arabia. To achieve this goal, two objectives were identified, to detect the prevalence of MEs in 2016 and to describe factors associated with MEs.

\section{Methods}

This is a quantitative study that utilized a retrospective cross-sectional design conducted in 2016 in tertiary care hospitals in the Al-Qassim region, Kingdom of Saudi Arabia. Approval was obtained from the Research and Ethical Committee of the tertiary hospitals (maternity and children's hospitals) in 2019 with IRB number 1440-1676342.

Medical error was the main variable for the analysis and was identified using two methods. The first involved examining a 2016 report on hospital-based incidents of MEs where it is mandatory for health-care staff to report MEs. The second method relied on two pharmacists to manually review written prescriptions from January 2016 to December 2016.

All medical charts and incident reports in 2016 were reviewed for MEs. On identifying an incidence of $\mathrm{ME}$, the reviewer classified the ME by severity, type, and stage of error. The definitions of ME severity were based on Neville's classification: Type A (potentially life threatening), type $B$ (non-life threatening), and type $C$ (minor nuisance or trivial) [4]. The error description type was partially taken from the National Coordinating Council for ME Reporting and Prevention [11]. The stages of error included prescribing, preparing, dispensing, transcribing, administering, and monitoring [12].

Medical charts and incident reports were used to identify other factors (independent variables) such as those related to patients, hospital, and/or treatment as well as error descriptions. These factors included patient's age, health-care specialty associated with the error, time, route of administration, type of package container, and department source. Hospital departments were classified according to the studied hospital and were divided into neonatal intensive care unit, pediatric intensive care unit, pediatric medical ward, critical care fetal unit, high dependency unit, operating room, pediatric health organizations, pediatric emergency room, obstetrics and gynecology (OB-GYN) emergency room, pediatric surgery ward, gynecology, OB-GYN ward, delivery room, and outpatient department (OPD).

Data comprised a series of descriptive statistics and trend frequencies of MEs for each month in 2016. All parametric statistical analyses were conducted using RStudio: Integrated Development for R (RStudio, Inc., Boston, MA). The threshold of significant was sat at value, $p<0.01$

\section{Results}

During the study period, 2123 MEs were reported for 213,489 prescriptions. The data recorded for the study population included age of patient, source of ME, time of error, route of administration, package container, outcome of error, department source, type of error, and stage involved. Table 1 presents MEs by patient age. The greatest number of prescription errors was associated with adult patients aged $18-40$ years $(46.82 \%)$ followed by patients aged 1 month-6 years (35.94\%).

\section{Table 1: Patient factors}

\begin{tabular}{lll}
\hline Factor & Frequency $(\%)$ & p-value \\
\hline Age of patient associated with ME & & $<0.0001$ \\
$1-30$ days & $70(3.30)$ & \\
1 month-6 years & $763(35.94)$ & \\
$6-12$ years & $193(9.09)$ & \\
$12-18$ years & $29(1.37)$ & \\
$18-40$ years & $994(46.82)$ & \\
40 years or older & $74(3.48)$ & \\
\hline
\end{tabular}

Table 2 lists MEs' frequency by associated healthcare specialty, time, and department. MEs most commonly originated with physicians (1282; 60.38\%) followed by nurses $(755 ; 35.58 \%)$ and then pharmacists $(86 ; 4.05 \%$; $p$ $<0.0001)$. The rate of MEs was higher for morning shifts, at $1837(86.53 \%)$, versus night shifts, at $21(0.79 \%)$. Most observed MEs occurred in the OPD $(905 ; 42.45 \%)$ followed by the obstetric-gynecological ward (407; 19.09\%).

Table 2: Hospital factors

\begin{tabular}{lll}
\hline Factor & Frequency (\%) & p-value \\
\hline Health-care specialty associated with ME & & $<0.0001$ \\
Physician & $1282(60.38)$ & \\
Nurses & $755(35.58)$ & \\
Pharmacist & $86(4.05)$ & $<0.0001$ \\
Time of ME & & \\
Morning & $1837(86.53)$ & \\
Afternoon & $256(12.54)$ & \\
Night & $21(0.79)$ & \\
Department* & & \\
OPD & $905(42.45)$ & \\
OBW & $407(19.09)$ & \\
GYNE & $192(9.01)$ & \\
PMW & $180(8.44)$ & \\
P-ER & $96(4.50)$ & \\
OB-ER & $72(3.38)$ & \\
NICU & $63(2.95)$ & \\
Pharmacy inpatient & $55(2.58)$ & \\
Pharmacy OPD & $41(1.92)$ & \\
PICU & $31(1.45)$ & \\
Pharmacy IV/TPN & $27(1.27)$ & \\
PSW & $20(0.94)$ & \\
OR & $14(0.66)$ & \\
PHO & $13(0.61)$ & \\
HDU & $13(0.61)$ & \\
CCFU & $3(0.14)$ & \\
\hline${ }^{*}$ Multiple departments might contribute to a single error. NICU: Neonatal intensive care unit. PICU: Pediatric \\
intensive care unit, PMW: Pediatric medical ward, CCFU: Critical care fetal unit, HDU: High dependency \\
unit, OR: Operating room, PHO: Pediatric health organizations, PSW: Pediatric surgery ward, \\
Gyne: Gynecology, OPD: Outpatient department. & & \\
& &
\end{tabular}


Out of the 2123 errors, prescribing errors were the most frequently observed in the prescribing stage; the other stages are summarized in Table 3. Categorization by route of administration reveals that the IV route was the most commonly involved (1143; $53.84 \%)$ followed by the oral route $(761 ; 35.85 \%)$. Similarly, single dose, vial, and ampoule were the most common sources of MEs (1149; $54.04 \%)$ followed by unit dose and oral $(727 ; 34.21 \%)$.

Table 3: Treatment factors

\begin{tabular}{lll}
\hline Factor & Frequency (\%) & p-value \\
\hline Stage & $1271(59.87)$ & $<0.0001$ \\
Prescribing & $732(34.48)$ & \\
Administration & $84(3.95)$ & \\
Dispensing & $33(1.55)$ & \\
Transcribing & $3(0.14)$ & $<0.0001$ \\
Monitoring & & \\
Route of administration & $1143(53.84)$ & \\
IV & $761(35.85)$ & \\
Oral & $170(8.00)$ & \\
SC & $49(2.31)$ & \\
Others & $1149(54.04)$ & \\
Package container & $727(34.21)$ & \\
Single dose/vial/ampoule & $113(5.36)$ & \\
Unit dose/oral & $68(3.24)$ & \\
Syringe & $65(3.10)$ & \\
Bottle & $1(0.05)$ & \\
IV piggyback & & \\
Intravenous solution & &
\end{tabular}

Analysis of the outcome of error types (Table 4) revealed that error types $B$ and $C$ were the most common, with only few type A errors identified $(0.14 \%)$. For the categories of prescription MEs identified, the most common error was incomplete data $(34.27 \%)$ followed by prescription in illegible handwriting $(14.88 \%)$. The least common ME was prescription of the wrong strength $(0.17 \%)$. Trend of MEs during 2016 year by month was condcuted. Total of prescriptions, Total ME reports and percentage of MEs reports were collected (Table 5).

\section{Table 4: Error descriptions}

\begin{tabular}{ll}
\hline Factor & Frequency (\%) \\
\hline Outcome of error* & \\
A (potential risk) & $3(0.14)$ \\
B (near miss) & $1733(81.63)$ \\
C & $387(18.23)$ \\
Type of error \$ & \\
Incomplete data & $790(34.27)$ \\
Illegible handwriting & $343(14.88)$ \\
Dose omission & $302(13.10)$ \\
Improper dose & $225(9.76)$ \\
No double check & $159(6.89)$ \\
Wrong frequency & $159(6.89)$ \\
Wrong abbreviation & $88(3.82)$ \\
Wrong duration & $61(2.64)$ \\
Drug-drug interaction & $33(1.43)$ \\
Wrong time of administration & $25(1.08)$ \\
Pregnancy risk drug (category C) & $23(0.99)$ \\
Therapeutic duplication & $20(0.86)$ \\
Given without documentation & $18(0.78)$ \\
No label & $13(0.56)$ \\
Wrong drug & $10(0.43)$ \\
Wrong drug preparation & $10(0.43)$ \\
Wrong rate & $9(0.39)$ \\
Wrong route of admin & $9(0.39)$ \\
Wrong documentation & $4(0.17)$ \\
Wrong strength & $4(0.17)$ \\
\hline${ }^{*}$ No errors reported with outcomes classified as D, E, F, G, H, and I. ${ }^{5}$ A single prescription might contain \\
multiple errors.
\end{tabular}

\section{Discussion}

This study was designed to investigate of MEs, particularly to identify, analyze, and compare common types of medication prescription errors, and the factors associated with the root causes of these errors, in a large tertiary hospital in the Qassim region. It revealed that most MEs were associated with adult patients, in line with the findings of Zakharov et al. (2012) who reported that about half of cases associated with MEs in a local hospital were adults [13]. This is not surprising, considering that adult patients have more medical prescriptions than younger and older patients [14].

Although we found that MEs most commonly originated with physicians followed by nurses and then pharmacists, no consensus is present in the literature. On the one hand, Neyaz et al. (2011) reported that physicians failed to provide information necessary for the safe dispensing and administration of drugs [1], but on the other hand, Wirtz et al. (2003) reported that a third of MEs were associated with nurses [15]. Motluk (2018), notably, suggested that burnout is the main factor behind health-care providers' errors [16].

The majority of MEs occurred at morning and in the OPD, suggesting that health-care providers encounter high workloads in these particular settings - workload having been shown to be correlated with missed care in general [12]. Similarly, Saghafi et al. (2014) found that morning shifts in the outpatient clinic contributed to more than $50 \%$ of reported MEs [12].

Error types $\mathrm{B}$ and $\mathrm{C}$ were the most common, consistent with reports in the literature [12]. Our analysis highlighted incomplete data, illegible handwriting, and dose omission. All can lead to serious errors, and health-care practitioners should take an active role in minimizing these MEs. For instance, we found that IV injections or infusions were the route of administration most vulnerable to ME, due to their sensitive nature, with a small error in IV injection potentially leading to a fatal outcome [17]. Nurses who deal with IV medication must thus combine caution with knowledge of administration technique and packaging [17].

An error rate of $0.99 \%$ of prescriptions per month was detected, but the ratio of errors was higher during summer (June-August), increasing from $1.19 \%$ to $1.25 \%$. A review of other studies revealed that summer is marked by increase in mortality and morbidity and a decrease in therapeutic outcomes in teaching hospitals, being a time of year associated with physician changeover [18]. Health-care staffs are more likely to change workforce or go on vacation during

Table 5: Trend of MEs during 2016

\begin{tabular}{|c|c|c|c|c|c|c|c|c|c|c|c|c|c|}
\hline Category & January & February & March & April & May & June & July & August & September & October & November & December & Year \\
\hline Total prescriptions & 17,852 & 17,528 & 18,216 & 17,986 & 18,522 & 16,963 & 18,276 & 17,584 & 16,843 & 17,284 & 18,453 & 17,982 & 213,489 \\
\hline Total ME reports & 104 & 128 & 143 & 159 & 184 & 202 & 211 & 219 & 193 & 206 & 201 & 173 & 2123 \\
\hline$\%$ of ME reports ${ }^{*}$ & 0.58 & 0.73 & 0.78 & 0.88 & 0.99 & 1.19 & 1.15 & 1.25 & 1.15 & 1.19 & 1.09 & 0.96 & 0.99 \\
\hline
\end{tabular}


the summer, causing an association between summer breaks and low health-care outcomes that have been called the "July Effect" [19]. This study provides robust methodology in identifying MEs with a specific focus on certain categories. However, this study was conducted in a local hospital specialized in maternity and children's, therefore, the results only represented to similar hospitals.

\section{Conclusion}

Despite the low number of MEs recorded during the study period, some of the errors were indeed serious. Based on the findings of this study, policy-makers should consider strategies for increasing efficiency in the hospital setting.

\section{References}

1. Neyaz Y, Khoja T, Qureshi NA, Magzoub MA, HaycoxA, Walley T. Medication prescribing pattern in primary care in Riyadh city, Saudi Arabia. East Mediterr Health J. 2011;17(2):149-54. https:// doi.org/10.26719/2011.17.2.132

PMid:21735950

2. Alqenae FA, Steinke D, Keers RN. Prevalence and nature of medication errors and medication-related harm following discharge from hospital to community settings: A systematic review. Drug Saf. 2020;125:259. https://doi.org/10.1007/ s40264-020-00918-3

PMid:32125666

3. Alsulami Z, Conroy S, Choonara I. Medication errors in the Middle East countries: A systematic review of the literature. Eur J Clin Pharmacol. 2013;69(4):995-1008. https://doi.org/10.1007/ s00228-012-1435-y

PMid:23090705

4. Khoja T, Neyaz Y, Qureshi NA, Magzoub MA, Haycox A, Walley T. (2011). Medication errors in primary care in Riyadh city, Saudi Arabia, 17(2).

5. Dyab EA, Elkalmi RM, Bux SH, Jamshed SQ. Exploration of nurses' knowledge, attitudes, and perceived barriers towards medication error reporting in a tertiary health care facility: A qualitative approach. Pharmacy (Basel). 2018;6(4):120. https://doi.org/10.3390/pharmacy6040120

PMid:30400619

6. Neville RG, Robertson F, Livingstone S, Crombie IK. A classification of prescription errors. J R Coll Gen Pract. 1989;39(320):110-2.

PMid:2555487

7. Alshaikh M, Mayet A, Aljadhey H. Medication error reporting in a university teaching hospital in Saudi Arabia. J Patient Saf. 2013;9(3):145-9. https://doi.org/10.1097/ pts.0b013e3182845044

PMid:23370218

8. Al-saleh KS, Ramadan MZ. Studying medical errors among hospital-staff at Saudi health providers. J Mater Sci Eng. 2012;2(1):41-52.

9. Al Khaja KA., Sequeira RP, Al-Ansari TM, Damanhori AH. Prescription writing skills of residents in a family practice residency programme in Bahrain. Postgrad Med J. 2008;84(990):198-204. https://doi.org/10.1136/pgmj.2007.062547 PMid: 18424577

10. Zakharov S, Tomas N, Pelclova D. Medication errors-an enduring problem for children and elderly patients. Ups J Med Sci. 2012;117(3):309-17. https://doi.org/10.3109/03009734.201 2.659771

PMid:22376241

11. Saghafi $F$, Zargarzadeh AH. Medication error detection in two major teaching hospitals: What are the types of errors? J Res Med Sci. 2014;19(7):617-23.

PMid:25364360

12. Gorgich EA, Barfroshan S, Ghoreishi G, Yaghoobi M Investigating the causes of medication errors and strategies to prevention of them from nurses and nursing student viewpoint. Glob J Health Sci. 2016;8(8):54448. https://doi.org/10.5539/ gjhs.v8n8p220 PMid:27045413

13. Zhou S, Kang H, Yao B, Gong Y. Analyzing medication error reports in clinical settings: An automated pipeline approach. AMIA Annu Symp Proc. 2018;2018:1611-20. PMid:30815207

14. Seden K, Kirkham JJ, Kennedy T, Lloyd M, James S, McManus A, et al. Cross-sectional study of prescribing errors in patients admitted to nine hospitals across North West England. BMJ Open. 2013;3(1):1-14. https://doi.org/10.1136/ bmjopen-2012-002036

15. Salmasi S, Khan TM, Hong YH, Ming LC, Wong TW. Medication errors in the Southeast Asian countries: A systematic review. PLoS One. 2015;10(9):e0136545. https://doi.org/10.1371/ journal.pone. 0136545 PMid:26340679

16. Jhanjee A, Bhatia M, Oberoi A, Srivastava S. Medication errors in psychiatric practice-a cross-sectional study. Delhi Psychiatry J. 2012;15(1):5-13.

17. Cheragi MA, Manoocheri H, Mohammadnejad E, Ehsani SR. Types and causes of medication errors from nurse's viewpoint. Iran J Nurs Midwifery Res. 2013;18(3):228-31. PMid:23983760

18. Keers RN, Williams SD, Cooke J, Ashcroft DM. Causes of medication administration errors in hospitals: A systematic review of quantitative and qualitative evidence. Drug Saf. 2013;36(11):1045-67. https://doi.org/10.1007/s40264-013-0090-2 PMid:23975331

19. Ravi P, Trinh VQ, Sun M, Sammon J, Sukumar S, Gervais MK et al. Is there any evidence of a "July effect" in patients undergoing major cancer surgery? Can J Surg. 2014;57(2):82-8. https://doi.org/10.1503/cjs.002713

PMid:24666444 